\title{
Open problems in commutative ring theory
}

\author{
Paul-Jean Cahen $*$ Marco Fontana $\stackrel{\dagger}{\dagger}$ Sophie Frisch ${ }^{\ddagger}$ and Sarah Glaz ${ }^{\S}$
}

December 23, 2013

\begin{abstract}
This article consists of a collection of open problems in commutative algebra. The collection covers a wide range of topics from both Noetherian and non-Noetherian ring theory and exhibits a variety of research approaches, including the use of homological algebra, ring theoretic methods, and star and semistar operation techniques. The problems were contributed by the authors and editors of this volume, as well as other researchers in the area.

Keywords: Prüfer ring, homological dimensions, integral closure, group ring, grade, complete ring, McCoy ring, Straight domain, divided domain, integer valued polynomials, factorial, density, matrix ring, overring, absorbing ideal, Kronecker function ring, stable ring, divisorial domain, Mori domain, finite character, $\mathrm{P} v \mathrm{MD}$, semistar operation, star operation, Jaffard domain, locally tame domain, factorization, spectrum of a ring, integral closure of an ideal, Rees algebra, Rees valuation.

Mthematics Subject Classification (2010): 13-02; 13A05; 13A15; 13A18; 13B22; 13C15; 13D05; 13D99; 13E05; 13F05; 13F20; 13F30; $13 \mathrm{G} 05$
\end{abstract}

\section{Introduction}

This article consists of a collection of open problems in commutative algebra. The collection covers a wide range of topics from both Noetherian and nonNoetherian ring theory and exhibits a variety of research approaches, including

${ }^{*}$ Paul-Jean Cahen (Corresponding author), 12 Traverse du Lavoir de Grand-Mère, 13100 Aix en Provence, France. e-mail: pauljean.cahen@gmail.com

${ }^{\dagger}$ Marco Fontana, Dipartimento di Matematica e Fisica, Università degli Studi Roma Tre, Largo San Leonardo Murialdo 1, 00146 Roma, Italy. e-mail: fontana@mat.uniroma3.it

${ }^{\ddagger}$ Sophie Frisch, Mathematics Department, Graz University of Technology, Steyrergasse 30, 8010 Graz, Austria. e-mail: frisch@tugraz.at

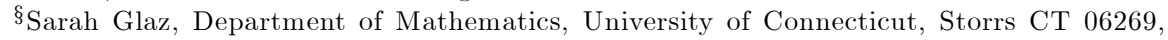
USA.e-mail: Sarah.Glaz@uconn.edu 
the use of homological algebra, ring theoretic methods, and star and semistar operation techniques. The problems were sent to us, in response to our request, by the authors of the articles in this volume and several other researchers in the area. We also included our own contributions. Some of these problems appear in other articles in this volume, while others are unrelated to any of the articles, but were considered important by their proposers. The problems were gathered by the contributors from a variety of sources, and include long standing conjectures as well as questions that were generated by the most recent research in the field. Definitions and clarifying comments were added to make the problems more self contained, but, as a rule, if unidentified notions are used the reader can find the relevant definitions in the cited references. The purpose of this article is to generate research, and we hope that the problems proposed here will keep researchers happily busy for many years.

The underlying assumption is that all rings are commutative with 1 (and $1 \neq 0$ ), all modules are unital, all groups are abelian, and the term "local ring" refers to a not-necessarily Noetherian ring with a unique maximal ideal. Several notions and ring constructions appear in a number of the proposed problems. For the reader's convenience, we mention a few definitions and sources of information here:

Let $R$ be a commutative ring and let $G$ be an abelian group written multiplicatively. The group ring $R G$ is the free $R$ module on the elements of $G$ with multiplication induced by $G$. An element $x$ in $R G$ has a unique expression

$x=\sum_{g \epsilon G} x_{g} g$, where $x_{g} \epsilon R$ and all but finitely many $x_{g}$ are zero. Addition and multiplication in $R G$ are defined analogously to the standard polynomial operations. Basic information about commutative group rings may be found in [60, 84] and [63, Chapter 8 (Section 2)].

Let $D$ be a domain with quotient field $K, \operatorname{Int}(D)$ denotes the ring of integervalued polynomials, that is, $\operatorname{Int}(D)=\{f \in K[X] \mid f(D) \subseteq D\}$. More generally, for a subset $E$ of $K, \operatorname{Int}(E, D)=\{f \in K[X] \mid f(E) \subseteq D\}$, and thus, $\operatorname{Int}(D)=$ $\operatorname{Int}(D, D)$. For several indeterminates, $\operatorname{Int}\left(D^{n}\right)=\left\{f \in K\left[X_{1}, \ldots, X_{n}\right] \mid f\left(D^{n}\right) \subseteq\right.$ $D\}$. For a $D$-algebra $A$, containing $D, \operatorname{Int}_{K}(A)=\{f \in K[X] \mid f(A) \subseteq A\}$. Note that $\operatorname{Int}_{K}(A)$ is contained in $\operatorname{Int}(D)$ if and only if $A \cap K=D$. Basic information about integer valued polynomials may be found in [19].

Basic information on the star and semistar operations that appear in some of these problems may be found in [59, Sections 32 and 34] and [95].

Basic information on the integral closure of ideals in Noetherian rings that is used in some of these problems may be found in [81].

Finally, for interested readers, we provide the list of contributors in the Acknowledgment section at the end of this article.

\section{Open Problems}

\section{Problem 1}

Glaz [67] and Bazzoni \& Glaz [14] consider, among other properties, the finitistic and weak global dimensions of rings satisfying various Prüfer conditions. 
The Prüfer conditions under considerations are:

(1) $R$ is semihereditary (i.e. finitely generated ideals of $R$ are projective).

(2) w.dim $R \leq 1$.

(3) $R$ is arithmetical (i.e. ideals of $R_{m}$ are totally ordered by inclusion for all maximal ideals $m$ of $R$ ).

(4) $R$ is Gaussian (i.e. $c(f g)=c(f) c(g)$ for all $f, g \in R[x]$ ).

(5) $R$ is locally Prüfer (i.e. $R_{p}$ is Prüfer for every prime ideal $p$ of $R$ ).

(6) $R$ is Prüfer (i.e. every finitely generated regular ideal of $R$ is invertible).

Let $\bmod R$ be the set of all $R$-modules admitting a projective resolution consisting of finitely generated projective modules. The finitistic projective dimension of $R$, is defined as: fp.dim $R=\sup \left\{\right.$ proj.dim $\operatorname{dim}_{R} M: M \epsilon \bmod R$ and proj.dim $\left.\operatorname{dim}_{R} M<\infty\right\}$. In general, $\operatorname{fp} \cdot \operatorname{dim} R \leq \mathrm{w} \cdot \operatorname{dim} R$, and if $R$ is a local coherent regular ring, then fp.dim $R=$ w.dim $R$ [67, Lemma 3.1]:

Problem 1a. Let $R$ be a Prüfer ring. Is fp.dim $R \leq 1$ ?

The answer is affirmative for Gaussian rings [67, Theorem 3.2 and 14, Proposition 5.3]. It is also clearly true for Prüfer domains.

Problem 1b. Let $R$ be a total ring of quotients. Is fp.dim $R=0$ ?

Note that a total ring of quotients is always a Prüfer ring, so this question asks if for this particular kind of Prüfer ring, fp.dim $R$ is not only at most equal to 1 , but is actually equal to 0 . This is true for a local Gaussian total ring of quotients [67, Theorem 3.2 (Case 1)]. More information and a detailed bibliography on the subject may be found in [68].

\section{Problem 2}

Using the results obtained for the finitistic projective dimension of rings (see previous problem) with various Prüfer conditions, it is possible to determine the values of the weak global dimensions of rings under certain Prüfer conditions $[14,67]$, but many questions are not yet answered:

Problem 2a. If $R$ is a Gaussian ring, then is w.dim $R=0,1$, or $\infty$ ?

This is the case for coherent Gaussian rings [67, Theorem 3.3] (and actually, more generally, for coherent Prüfer rings [14, Proposition 6.1]), arithmetical rings [97 and 14, remark in the last paragraph], and a particular case of Gaussian rings [14, Theorem 6.4].

Problem 2b. If $R$ is a total ring of quotients, is w.dim $R=0,1$, or $\infty$ ?

This is true if $R$ is coherent [68, Corollary 6.7], in which case w.dim $R=0$ or $\infty$; and also holds for one example of a non coherent ring [68, Example 6.8].

In general:

Problem 2c. What are the values of w.dim $R$ when $R$ is a Prüfer ring?

Additional information and references on the subject may be found in [68].

\section{Problem 3}

Is the integral closure of a one dimensional coherent domain in its field of quotients a Prüfer domain? 
This question, which also appears in [23, Problem 65], was posed by Vasconcelos. It had been answered positively in many, but not all, cases. A useful reference that will lead to many other useful references is [63, Chapter 5 (Section 3) and Chapter 7 (Section 4)].

\section{Problem 4}

A ring $R$ is a finite conductor ring if $a R \cap b R$ and $(0: c)$ are finitely generated ideals of $R$ for all elements $a, b$, and $c$ in $R$. A ring $R$ is a quasi coherent ring if $a_{1} R \cap \ldots \cap a_{n} R$ and $(0: c)$ are finitely generated ideals of $R$ for all elements $a_{1}, \ldots, a_{n}$ and $c$ in $R$. Examples of both classes of rings include all coherent rings, UFDs, GCD domains, G-GCD domains (that is, domains in which the intersection of two invertible ideals is an invertible ideal), and the still more general G-GCD rings (that is, rings in which principal ideals are projective and the intersection of two finitely generated flat ideals is a finitely generated flat ideal). For more information on these classes of rings see references [64, 65]. Let $G$ be a multiplicative abelian group and let $R G$ be the group ring of $G$ over $R$. In the group ring setting where $R$ is a domain, characterizations of group rings as UFDs and GCD domains were obtained in [61, Theorems 6.1, 6.4, and 7.17]. In the case where $R$ is a ring with zero divisors, however, the behavior of the finite conductor and quasi coherent properties has been only partial described. Specifically, in the general ring setting, both properties descend from $R G$ to $R$ [65, Proposition 3.2], and the question of ascent from $R$ to $R G$ reduces to the situation where $G$ is finitely generated [65, Proposition 3.1]. This, however, does not solve the problem of ascent for either property. Even in the case where $R$ is a G-GCD ring and $G$ is an infinite cyclic group, ascent is unknown.

Problem 4a. Assume that $R$ is a G-GCD ring and $G$ is a finitely generated abelian group. Do the finite conductor and the quasi coherent properties ascend from $R$ to $R G$ ?

Further explorations of these conditions in the group ring setting may shed light on a more general problem:

Problem 4b. Are the finite conductor and quasi coherent properties for rings distinct?

Other useful references include [51] and [69].

\section{Problem 5}

Let $R$ be a commutative ring and let $G$ be an abelian group with the property that the order of every element of $G$ is invertible in $R$. Then w.dim $R G=$ w.dim $R+\operatorname{rank} G[33$, Theorem] and [62, Theorem 2]. With the aid of this formula, it is possible to characterize von Neumann regular and semihereditary group rings $R G$ [62, Corollaries 1 and 2].

Problem 5a. Is there a similar formula that relates the global dimension of $R G$ to the global dimension of $R$ in combination with some invariant of the group $G$ ?

In this direction, there is one classical result, Maschke's Theorem [84, page 47], which pertains to a special case of semisimple rings (that is, rings of global 
dimension zero): Let $G$ be a finite group and let $k$ be a field. Then $k G$ is a semisimple ring if and only if the characteristic of $k$ does not divide the order of the group $G$. Beyond this, characterizations of group rings of finite global dimension are not known, even for the simple cases of global dimension zero (semisimple rings) or one (hereditary rings).

Problem 5b. Find characterizations of semisimple and hereditary group rings.

Other useful references include [69, 103].

\section{Problem 6}

The generalization of the notion of a Cohen-Macaulay and a Gorenstein ring from the Noetherian to the non-Noetherian context is a recent development. As such, the questions of when a group ring $R G$, where $R$ is a commutative ring and $G$ is an abelian group, is Cohen-Macaulay or Gorenstein have yet to be investigated. The articles [72] and [80] introduce the notions of non-Noetherian Cohen-Macaulay and non-Noetherian Gorenstein, respectively. An excellent survey of these theories which discusses the underlying homological framework is found in [79].

\section{Problem 7}

This problem arises within the context of work done by Hamilton and Marley [72] to characterize non-Noetherian Cohen-Macaulay rings, as well as work of Hummel and Marley [80] to characterize non-Noetherian Gorenstein rings. In light of the role that homological dimensions, infinite finite free resolutions of modules, and local (co)homology, played in the development of these theories for local coherent rings, Hummel [79] posed the question:

Is there a non-Noetherian characterization of local complete intersection rings such that (coherent) local Gorenstein rings are complete intersections, and such that (coherent) local complete intersection rings are Cohen-Macaulay?

André [10] provided a characterization of non-Noetherian complete intersection rings parallel to its following Noetherian counterpart using André-Quillen homology: Let $(R, m)$ be a local Noetherian ring with residue field $k$, and let

$\beta_{i}=\operatorname{dim}_{k} \operatorname{Tor}_{i}^{R}(k, k)$ be the ith Betti number. $R$ is a complete intersection if and only if the Betti numbers appear in the following equality of power series: $\sum \beta_{i} x^{i}=\frac{(1+x)^{r}}{\left(1-x^{2}\right)^{s}}$ with $0<r-s=\operatorname{dim} R$.

While able to prove a similar characterization for non-Noetherian rings, André showed there was no relation between the integers $r$ and $s$ above in the non-Noetherian case.

In [79] one can find more background on this problem, as well as potential definitions or characterizations of non-Noetherian complete intersection rings.

\section{Problem 8}

Let $(R, M)$ be a Noetherian local ring. $R$ is said to be quasi-complete if for any decreasing sequence $\left\{A_{n}\right\}_{n=1}^{\infty}$ of ideals of $R$ and each natural number $k$, there exists a natural number $s_{k}$ with $A_{s_{k}} \subseteq\left(\bigcap_{n=1}^{\infty} A_{n}\right)+M^{k}$. If 
this condition holds for any decreasing sequence $\left\{A_{n}\right\}_{n=1}^{\infty}$ of ideals of $R$ with $\bigcap_{n=1}^{\infty} A_{n}=0$, then $R$ is called weakly quasi-complete (in which case, we actually have $A_{s_{k}} \subseteq M^{k}$ ). Now, if $R$ is complete, then $R$ is quasi-complete, which implies that $R$ is weakly quasi-complete. Also $R$ is quasi-complete if and only if each homomorphic image of $R$ is weakly quasi-complete. The implication " $R$ complete implies that $R$ is quasi-complete" was first proved by Chevalley [24, Lemma 7]; for a proof in this volume see [4, Theorem 1.3]. Note that a DVR is quasi-complete, but need not be complete. More generally, a one-dimensional Noetherian local domain is (weakly) quasi-complete if and only if it is analytically irreducible [4, Corollary 2.2].

Problem 8a. Is a weakly quasi-complete ring quasi-complete?

Problem 8 b. Let $k$ be a field and $R=k\left[X_{1}, \ldots, X_{n}\right]_{\left(X_{1}, \ldots, X_{n}\right)}, n \geq 2$. Is $R$ (weakly) quasi-complete?

Regarding problem $8 \mathrm{~b}$, in [4, Conjecture 1] it is conjectured that $R$ is not weakly quasi-complete and in [4, Example 2.1] this is shown to be the case if $k$ is countable. Note that $R$ not being quasi-complete is equivalent to the existence of a height-one prime ideal $P$ of $k\left[\left[X_{1}, \ldots, X_{n}\right]\right]$ with $P \cap k\left[X_{1}, \ldots, X_{n}\right]=0$. For additional information see [4].

\section{Problem 9}

A commutative ring $R$ is said to be a McCoy ring if each finitely generated ideal $I \subseteq Z(R)$ (where $Z(R)$ denotes the set of zero divisors of $R$ ) has a nonzero annihilator. In 1980, Akiba proved that if $R$ is an integrally closed reduced McCoy ring, then the polynomial ring $R[X]$ is also integrally closed $[2$, Theorem $3.2]$. He also proved that if $R_{M}$ is an integrally closed domain for each maximal ideal $M$, then $R[X]$ is integrally closed [2, Corollary 1.3]. In addition he provided an example of a reduced ring $R$ that is not a McCoy ring but locally is an integrally closed domain [2, Example]. Combining his results, one has that if $R$ is a reduced ring such that $R_{M}$ is an integrally closed McCoy ring for each maximal ideal $M$, then $R[X]$ is integrally closed [78, page 103].

Does there exist an integrally closed reduced ring $R$ such that $R_{M}$ is an integrally closed McCoy ring for each maximal ideal $M$, but $R$ is not a McCoy ring and it is not locally a domain?

\section{Problem 10}

With the notation and definitions of the previous problem, a ring $R$ has $\left(A_{n}\right)$ if each ideal $I \subseteq Z(R)$ that can be generated by $n$ (or fewer) elements has a nonzero annihilator. Example 2.5 in [90], shows that for each $n \geq 2$, there are reduced rings which have $\left(A_{n}\right)$ but not $\left(A_{n+1}\right)$. An alternate restriction on zero divisors is (a.c.): $R$ has (a.c.) if for each pair of elements $r, s \in R$, there is an element $t \in R$ such that $\operatorname{Ann}(r, s)=A n n(t)$. The rings in Examples 2.2 and 2.4 of [90] show that there are reduced McCoy rings that do not have (a.c.), and reduced rings with (a.c.) that are not McCoy.

Do there exist reduced rings that have both (a.c.) and $\left(A_{n}\right)$ for some $n \geq 2$ that are not McCoy rings? 


\section{Problem 11}

Let $P$ be a nonzero prime ideal of a Prüfer domain $R$. Then, it is known that $P S$ is a divisorial prime in each overring $R \subseteq S \subseteq R_{P}$ if and only if $P R_{P}$ is principal and each $P$-primary ideal (of $R$ ) is a divisorial ideal of $R$.

Problem 11a. Characterize when $P$ (and $R$ ) is such that $P \cap T$ is a divisorial prime of $T$ for each Prüfer domain $T \subseteq R$ with the same quotient field as $R$.

Note that no assumption has been made about $P S$ being, or not being, divisorial for $R \subsetneq S \subseteq R_{P}$. This is true for each $P$ if $\mathbb{Z} \subseteq R \subseteq \mathbb{Q}$.

Problem 11b. Characterize when $P$ (and $R$ ) is such that there is no Prüfer domain $T \subseteq R$ with the same quotient field as $R$ such that $P \cap T$ is a divisorial prime of $T$.

More information about the problem may be found in [42].

\section{Problem 12}

Let $D$ be an almost Dedekind domain with a non-invertible maximal ideal $M$. Then $D(X)$ is also an almost Dedekind domain and $M D(X)$ is a non-invertible maximal ideal with corresponding residue field $F(X)$ where $F=D / M$. Let $R$ be the pullback of $F[X]_{(X)}$ over $M D(X)$. Then $R$ is a Prüfer domain, $R \subsetneq$ $D(X) \subsetneq D(X)_{M D(X)}, M D(X)$ is a divisorial prime ideal of $R$, it is not a divisorial (prime) ideal of $D(X)$ but $M D(X)_{M D(X)}$ is a divisorial prime ideal of $D(X)_{M D(X)}$. In contrast, if $P$ is a nonzero non-maximal prime of the ring of entire functions $E$ and $N$ is a maximal ideal that contains $P$, then $E \subsetneq E_{N} \subsetneq$ $E_{P}, P\left(=P^{2}\right)$ is not a divisorial ideal of $E, P E_{N}$ is a divisorial prime ideal of $E_{N}$ and $P E_{P}$ is not a divisorial (prime) ideal of $E_{P}$.

Problem 12a. Does there exist a Prüfer domain $R$ with a nonzero prime $P$ such that there is a countably infinite chain of overrings $R=R_{0} \subsetneq R_{1} \subsetneq R_{2} \subsetneq$ $\cdots \subsetneq R_{P}$ where for all $n \geq 0, P R_{2 n}$ is a divisorial ideal of $R_{2 n}$ and $P R_{2 n+1}$ is an ideal of $R_{2 n+1}$ that is not divisorial? If such a chain exists, is $P S$ divisorial or not divisorial as an ideal of $S=\bigcup R_{m}\left(\subseteq R_{P}\right)$ ?

Problem 12b. Does there exist a Prüfer domain $R$ with a nonzero prime $P$ such that there is a countably infinite chain of underrings $R=R_{0} \supsetneq R_{1} \supsetneq$ $R_{2} \supsetneq \cdots \supsetneq T=\bigcap R_{m}$ with $T$ having the same quotient field as $R$ where for all $n \geq 0, P \cap R_{2 n}$ is a divisorial ideal of $R_{2 n}$ and $P \cap R_{2 n+1}$ is not a divisorial ideal of $R_{2 n+1}$ ? If such a chain exists, is $P \cap T$ divisorial or not divisorial as an ideal of $T$ ?

More information about the problem may be found in [42].

\section{Problem 13}

A domain $D$ with field of quotients $K$ is called a straight domain if for every overring $S$ of $D, S / P S$ is torsion free over $D / P$, for every prime ideal $P$ of $D$.

Problem 13a. If $R_{P}$ and $R / P$ are straight domains does this imply that $R+P R_{P}$ is a straight domain?

A domain $D$ is called divided if $P D_{P}=P$ for every prime ideal $P$ of $D ; D$ is called locally divided if $D_{M}$ is divided for every maximal ideal $M$ of $D$. An answer to question 13a may shed light on an open question posed in [31]: 
Problem 13b. Does there exist a straight domain which is not locally divided?

Extending these definitions, analogously, to general rings, we note that there exists a straight ring which is not a domain and not locally divided. For more details, see [32, Section 2].

\section{Problem 14}

Comparing the Krull dimensions of the ring $\operatorname{Int}(D)$ of integer-valued polynomials and of the classical ring $D[X]$ of polynomials with coefficients in $D$, it is known that $\operatorname{dim}(\operatorname{Int}(D)) \geq \operatorname{dim}(D[X])-1$, with possibility of equality [19, Example V.1.12]. The question of an upper bound remains open: It is conjectured to be equal to $\operatorname{dim}(D[X])$.

\section{Problem 15}

Considering a one-dimensional local Noetherian domain $D$ with finite residue field, when does $\operatorname{Int}(D)$ satisfy the almost strong Skolem property?

See [19, Chapter VII] for a survey of Skolem properties. It is known that it is enough that $D$ be analytically irreducible, and was recently shown that $D$ must be unibranched [20] leaving open the question of a necessary and sufficient condition.

\section{Problem 16}

A sequence $\left\{a_{n}\right\}_{n>0}$ of integers is said to be self (simultaneously) ordered if for all positive integers $m, n: \prod_{k=0}^{n-1}\left(a_{n}-a_{k}\right)$ divides $\prod_{k=0}^{n-1}\left(a_{m}-a_{k}\right)$. If $f$ is a non-constant polynomial of $\mathbb{Z}[X]$, distinct from $\pm X$, then, for every $x \in$ $\mathbb{Z}$, the sequence $\left\{f^{* n}(x)\right\}_{n>0}$ (where $f^{* n}$ denotes the $n$-th iterate of $f$ ) is self ordered [1, Proposition 18]. For instance, the sequence $\left\{q^{n}\right\}_{n \geq 0}$, with $q \neq 0, \pm 1$, obtained with $f(X)=q X$ and $x=1$, is self ordered. Aside from the (infinitely many) sequences obtained with such a dynamical construction, are the three following 'natural' sequences: $\left\{(-1)^{n}\left[\frac{n}{2}\right]\right\}_{n \geq 1},\left\{n^{2}\right\}_{n \geq 0}$, and $\left\{\frac{n(n+1)}{2}\right\}_{n \geq 0}$ Also, if the sequence $\left\{a_{n}\right\}_{n \geq 0}$ is self ordered, then so is $\left\{b a_{n}+c\right\}_{n \geq 0}$, for all integers $b \neq 0$ and $c$. We note the importance of the word 'natural' (although not defined), for instance, the 'natural candidates' $\left\{n^{2}\right\}_{n \geq 1}$ and $\left\{n^{k}\right\}_{n \geq 0}$ for $k \geq 3$ are not self ordered. On the other hand, one can construct infinitely many 'artificial' self ordered sequences by the following ad hoc construction: choose two distinct integers $a_{0}$ and $a_{1}$ and, for $n \geq 1$, define inductively $a_{n+1}$ to be any integer, distinct from the $a_{k}$ 's for $0 \leq k \leq n$, such that $\prod_{k=0}^{n-1}\left(a_{n}-a_{k}\right)$ divides $a_{n+1}-a_{0}$. These integers can be chosen to be prime numbers, thanks to Dirichlet's theorem, and hence, there are infinitely many self ordered sequences contained in the set $\mathbb{P}$ of prime numbers, although $\mathbb{P}$ itself cannot be self ordered. The question is:

Are there any other 'natural' self ordered sequences neither obtained by a dynamical construction nor by an affine map applied to the three previous examples? 
More details can be found in [22, Section 5.2, Q.2].

\section{Problem 17}

The $n^{t h}$ Bhargava factorial [15] associated to an infinite subset $E$ of $\mathbb{Z}$ is the integer $n !_{E}$ such that $\frac{1}{n !_{E}}$ is the generator of the fractional ideal formed by the leading coefficients of the polynomials $f(X) \in \operatorname{Int}(E, \mathbb{Z})$ with degree $\leq n[22$, Section 3]. These factorials have the following properties:

1. $0 !_{E}=1$

2. $\forall n \geq 0, n$ ! divides $n !_{E}$

3. $\forall n, m \geq 0, n !_{E} \times m !_{E}$ divides $(n+m) !_{E}$

Mingarelli [92] called abstract factorials a sequence $\left\{n !_{a}\right\}_{n>0}$ of positive integers satisfying these three properties. Clearly, such sequences are nondecreasing, but although there cannot be three consecutive equal terms [92, Lemma 8], one may have $k !_{a}=(k+1) !_{a}$ for infinitely many $k$ [92, Proposition 12].

Are there subsets $E$ of $\mathbb{Z}$ such that the sequence of Bhargava's factorials $\left\{n !_{E}\right\}_{n \geq 0}$ is not ultimately strictly increasing? [22, Section 4.3, Q.1].

Note that, for $E=\left\{n^{3} \mid n \geq 0\right\}$, one has $3 !_{E}=4 !_{E}=504$, yet the sequence $\left\{n !_{E}\right\}_{n \geq 0}$ is ultimately strictly increasing.

\section{Problem 18}

Given an abstract factorial $\left\{n !_{a}\right\}_{n \geq 0}$ as in the previous question, one may define a generalization of the constant $e$ [92, Definition 17] by: $e_{a}=\sum_{n \geq 0} \frac{1}{n !}$. For Bhargava's factorial associated to a subset $E$ one denotes this number by $e_{E}$. For example, if $E=\mathbb{N}^{(2)}=\left\{n^{2} \mid n \geq 0\right\}$, then $e_{\mathbb{N}^{(2)}}=e+\frac{1}{e}$. The constant $e_{a}$ is always irrational [92, Theorem 28].

For which infinite subset $E$ is $e_{E}$ a transcendental number? [22, Section 6.5].

\section{Problem 19}

$\operatorname{Int}(D)$ is known to be a free $D$ module if $D$ is a Dedekind domain. $T V$ domains, defined by Houston and Zafrullah in [77], are domains in which $t$ ideals coincide with $v$-ideals. $T V P v M D$ domains were extensively studied in [82]. For a Krull domain, or more generally for a TV $\mathrm{P} v \mathrm{MD}$ domain, $\operatorname{Int}(D)$ is known to be locally free, and hence a flat $D$ module [37].

Problem 19a. Is $\operatorname{Int}(D)$ a flat $D$ module for any domain $D$ ?

More generally:

Problem 19b. Is $\operatorname{Int}(D)$ a free $D$-module for any domain $D$ ?

\section{Problem 20}

Let $D$ be an integral domain. The canonical $D$-algebra homomorphism $\operatorname{Int}(D)^{\otimes_{D} n} \longrightarrow \operatorname{Int}\left(D^{n}\right)$ is known to be an isomorphism if $\operatorname{Int}(D)$ is free, or if $D$ is locally free and $\operatorname{Int}\left(D_{\mathfrak{m}}\right)=\operatorname{Int}(D)_{\mathfrak{m}}$ for every maximal ideal $\mathfrak{m}$ of $D$ [35].

Is this canonical morphism always injective? surjective? 


\section{Problem 21}

Let $D$ be an integral domain. Does $\operatorname{Int}(D)$ always have a unique structure of a $D$-D-biring such that the inclusion $D[X] \longrightarrow \operatorname{Int}(D)$ is a homomorphism of $D$ - $D$-birings?

This is the case if the canonical $D$-algebra homomorphism $\operatorname{Int}(D)^{\otimes_{D} n} \longrightarrow$ $\operatorname{Int}\left(D^{n}\right)$ is an isomorphism for all $n[36]$.

\section{Problem 22}

Let $D \subseteq A$ be an extension of domains. Let $B$ be the quotient field of $A$ and $\operatorname{Int}(A)=\{f \in B[X] \mid f(A) \subseteq A\}$ be the ring of integer-valued polynomials of $A$ and similarly $\operatorname{Int}\left(A^{n}\right)$ be the ring of integer-valued polynomials in several indeterminates. If $\operatorname{Int}\left(D^{n}\right) \subseteq \operatorname{Int}\left(A^{n}\right)$ for all positive integer $n$, then the extension $D \subseteq A$ is said to be almost polynomially complete [35].

If $\operatorname{Int}(D) \subseteq \operatorname{Int}(A)$, does it follow that the extension $D \subseteq A$ is almost polynomially complete?

\section{Problem 23}

Recall that, for a set $S$ of non-negative integers, the natural density $\delta(S)$ of $S$ is defined to be $\delta(S)=\lim _{n \rightarrow \infty} \frac{|\{a \in S: a<n\}|}{n}$, provided the limit exists.

Let $K$ be a number field and $\mathcal{O}_{K}$ be the corresponding ring of algebraic integers. Consider the natural density $\delta\left(\operatorname{Int}\left(\mathcal{O}_{K}\right)\right)$ of the set of non-negative integers $n$ such that $\operatorname{Int}_{n}\left(\mathcal{O}_{K}\right)$ is free $\left(\operatorname{Int}_{n}\left(\mathcal{O}_{K}\right)\right.$ denotes the $\mathcal{O}_{K}$-module formed by the integer-valued polynomials of degree at most $n$ ).

Prove or disprove the following conjecture [38]: $\delta\left(\operatorname{Int}\left(\mathcal{O}_{K}\right)\right)$ exists, is rational, and is at least $1 / \operatorname{Card}\left(\mathrm{PO}\left(\mathcal{O}_{K}\right)\right)$ (where $\mathrm{PO}\left(\mathcal{O}_{K}\right)$ ) denotes the Pólya group of $\left.\mathcal{O}_{K}\right)$. Then compute $\delta\left(\operatorname{Int}\left(\mathcal{O}_{K}\right)\right)$.

\section{Problem 24}

Consider the $\operatorname{ring} \operatorname{Int}(E, \mathbb{Z})$ where $E$ is the set formed by the elements of a sequence $\left\{u_{n}\right\}_{n \geq 0}$ of integers determined by a recursion $u_{n+1}=a u_{n}+b u_{n-1}$ and initial values $u_{0}, u_{1}$.

Problem 24a. Compute a regular basis (that is, a basis with $n$-th term of degree $n)$ of the $\mathbb{Z}$-module $\operatorname{Int}(E, \mathbb{Z})$.

Problem 24b. Compute the characteristic sequence of $\operatorname{Int}(E, \mathbb{Z})$ with respect to each prime $p$, that is, the sequence $\left\{\alpha_{p}(n)\right\}_{n \geq 0}$, where $\alpha_{p}(n)$ is the $p$-adic valuation of the fractional ideal consisting of 0 and the leading coefficients of elements of $\operatorname{Int}(E, \mathbb{Z})$ of degree no more than $n$.

Problem 24c. Determine the asymptotic behavior of this sequence, that is,

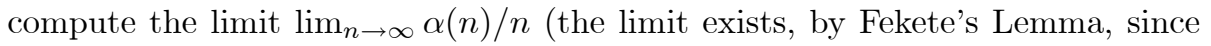
the sequence $\{\alpha(n)\}$ is superadditive).

Based on the results of Coelho and Parry [25] answers are known if $b= \pm 1$, which includes the cases of the Fibonacci and Lucas numbers [83, 102], however the method used there does not seem to extend to general $a, b$. This question can, of course, be extended to higher order recursive sequences. 


\section{Problem 25}

There has been much recent progress in understanding $\operatorname{Int}_{\mathbb{Q}}\left(M_{n}(\mathbb{Z})\right)$ where $M_{n}(\mathbb{Z})$ is the ring of $n \times n$ matrices with integer coefficients, beginning with Frisch's contribution [47] and more recently those of Peruginelli [98] and of Peruginelli and Werner [99]. These descriptions relate $\operatorname{Int}_{\mathbb{Q}}\left(M_{n}(\mathbb{Z})\right)$ to polynomials integer valued on algebraic integers or divisible by irreducibles modulo $d \mathbb{Z}[x]$. There remains however, as in the previous problem, to:

Problem 25a. Compute a regular basis of $\operatorname{Int}_{\mathbb{Q}}\left(M_{n}(\mathbb{Z})\right)$.

Problem 25b. Compute its characteristic sequence with respect to each prime $p$.

Problem 25c. Determine the asymptotic behavior of this sequence.

\section{Problem 26}

Let $D$ be an integral domain and let $A$ be a torsion-free $D$-algebra that is finitely generated as a $D$-module. We consider the $\operatorname{ring} \operatorname{Int}_{K}(A)$ of polynomials, with coefficients in the quotient field $K$ of $D$, that are integer-valued over $A$, that is, $\operatorname{Int}_{K}(A)=\{f \in K[X] \mid f(A) \subseteq A\}$. For every $a \in A$, we denote by $\mu_{a}(X) \in D[X]$ the minimal polynomial of $a$ over $D$. Each polynomial $f$ in the pullback $D[X]+\mu_{a}(X) K[X]$ is obviously such that $f(a) \in A$. Hence: $\bigcap_{a \in A}\left(D[X]+\mu_{a}(X) K[X]\right) \subseteq \operatorname{Int}_{K}(A)$.

For which algebras $A$ as above does this inclusion become an equality?

Note that equality holds for the $D$-algebra $A=M_{n}(D)$ of $n \times n$ matrices over $D$ [99, Remark 3.4]. The $\operatorname{ring} \operatorname{Int}_{K}\left(M_{n}(D)\right)$ has been studied in several places, see for instance [46] and [98].

\section{Problem 27}

Let $D$ be an integral domain with quotient field $K$. Let $A$ be a torsionfree $D$-algebra, containing $D$, finitely generated as a $D$-module, and such that $K \cap A=D$. Letting $B=K \otimes_{D} A$ (that is, the ring of fractions $\frac{a}{d}$ with $a \in A$ and $d \in D, d \neq 0)$, one can consider the set $\operatorname{Int}(A)=\{f \in B[X] \mid f(A) \subseteq$ $A$ \}. Working with polynomials in $B[X]$, one assumes that the indeterminate $X$ commutes with all elements of $B$ and that polynomials are evaluated with $X$ on the right, see [85] for more details on polynomials with non-commuting coefficients. Note that $\operatorname{Int}_{K}(A)=\operatorname{Int}(A) \cap K[X]$. The set $\operatorname{Int}(A)$ is always a left $A$-module, but it is not clear whether it has a ring structure when $A$ is not commutative. A sufficient condition for $\operatorname{Int}(A)$ to be a ring is that each element of $A$ can be written as a finite sum $\sum_{i} c_{i} u_{i}$, where each $u_{i}$ is a unit of $A$ and each $c_{i}$ is central in $B$ [106]. Examples of algebras that meet this condition include the matrix rings $M_{n}(D)$ and group rings $D G$ where $G$ is a finite group. Yet this condition is not necessary [107].

Problem 27a. Give an example of a $D$-algebra $A$ such that $\operatorname{Int}(A)$ is not a ring (possibly relaxing some of the conditions on $A$, for instance that $A$ is finitely generated as a $D$-module). 
Problem 27b. In [107], it is conjectured that $\operatorname{Int}(A)$ is always a ring when $D$ has finite residue rings. Prove this conjecture or give a counterexample in this case.

\section{Problem 28}

In [89], it is shown that if $A$ is the ring of integers of an algebraic number field, then $\operatorname{Int}_{\mathbb{Q}}(A)$ is Prüfer. More generally, let $D$ be an integral domain, let $K$ be its quotient field and let $A$ be a $D$-algebra. If $\operatorname{Int}_{K}(A)$ is a Prüfer domain contained in $\operatorname{Int}(D)$, then $\operatorname{Int}(D)$ must also be Prüfer, and hence $D$ must be integrally closed. This condition is not sufficient: $\operatorname{Int}_{K}(A)$ need not even be integrally closed and [99] gives some general theorems regarding the integral closure of $\operatorname{Int}_{K}(A)$.

Determine when this integral closure is Prüfer.

Note that Loper [87] determined such a criterion for the classical ring of integer-valued polynomials.

\section{Problem 29}

In the early 90s, J.D. Sally gave expository talks on the question of which rings lie between a Noetherian domain $D$ and its quotient field $F$ [74]. The manuscript [74] provides abundant evidence that when the dimension of $D$ is greater than one, the class of rings between $D$ and $F$ is rich in interesting Noetherian and non-Noetherian rings. A narrower problem, which remains open, is the following:

Describe the integrally closed rings between a two-dimensional Noetherian domain and its quotient field.

Work on this problem is surveyed in [96]. The evidence suggests that this class of rings is quite complicated. A framework for describing the integrally closed rings between $\mathbb{Z}[X]$ and $\mathbb{Q}[X]$ is given in [88].

\section{Problem 30}

Let $R$ be a ring and let $n$ be a positive integer. A proper ideal $I$ of $R$ is called an $n$-absorbing ideal if whenever the product $x_{1} x_{2} \cdots x_{n+1} \in I$ for $x_{1}, x_{2}, \ldots, x_{n+1} \in R$, then there are $n$ of the $x_{i}$ 's whose product is in $I$. Clearly, a 1-absorbing ideal is just a prime ideal. A proper ideal $I$ of $R$ is called a strongly $n$-absorbing ideal if whenever $I_{1} I_{2} \cdots I_{n+1} \subseteq I$ for ideals $I_{1}, I_{1}, \ldots, I_{n+1}$ of $R$, then there are $n$ of the ideals $I_{i}$ 's whose product is a subset of $I$. Obviously, a strongly $n$-absorbing ideal is a $n$-absorbing ideal.

Problem 30a. Let $I$ be an $n$-absorbing ideal of $R$. Is $I$ a strongly $n$ absorbing ideal of $R$ ?

Note that if $n=1$ the answer is obviously positive and if $n=2$ a positive answer is contained in [11, Theorem 2.13].

Problem 30b. Let $I$ be an $n$-absorbing ideal of $R$. Is $\operatorname{rad}(I)^{n} \subseteq I$ ?

Note that if $I$ is an $n$-absorbing ideal, then so is $\operatorname{rad}(I)$. For $n=2$, the positive answer to this question is proved in [11, Theorem 2.4]. 
Problem 30c. Let $I$ be an $n$-absorbing ideal of $R$. Is $I[X]$ an $n$-absorbing ideal of the polynomial ring $R[X]$ ?

Note that if $n=2$, then the answer is affirmative [8, Theorem 4.15].

\section{Problem 31}

Let $F / K$ be a transcendental field extension, and let $X$ be an indeterminate over $F$. By a result of Halter-Koch [70, Theorem 2.2] $A=\bigcap_{V} V(X)=$ $\bigcap_{V} V[X]_{M_{V}[X]}$, where $V$ ranges over the valuation rings of $F / K$ and $M_{V}$ is the maximal ideal of $V$, is a Bézout domain. However, $A$ is not the Kronecker function ring of a domain with quotient field $F$ (and hence does not arise directly from an e.a.b. star operation). Fabbri and Heubo-Kwegna [39] show this issue can be circumvented when $F=K\left(X_{0}, X_{1}, \ldots, X_{n}\right)$ is a purely transcendental extension of $K$ by introducing the notion of projective star operations, which are glued together from traditional star operations on affine subsets of projective $n$-space. The ring $A$ is then a "projective" Kronecker function ring of the projective analogue of the $b$-operation. More generally, e.a.b. projective star operations give rise to projective Kronecker function rings. The theory of projective star operations is worked out in [39] for projective $n$-space, but the following problem remains open:

Develop projective star operations for projective varieties (i.e., for the case where $F / K$ is a finitely generated field extension that need not be purely transcendental)?

\section{Problem 32}

Let $D$ be a domain and let $I$ be a nonzero ideal of $D$. Recall that $I$ is called stable if $I$ is invertible in its endomorphism ring $E(I)=(I: I)$, and the integral domain $D$ is called (finitely) stable if each nonzero (finitely generated) ideal is stable. As usual, the ideal $I$ is called divisorial if $I=I^{v}=(D:(D: I))$, and $D$ is called a divisorial domain if each nonzero ideal is divisorial. A Mori domain is a domain satisfying the ascending chain condition on divisorial ideals. Clearly Noetherian and Krull domains are Mori. It has been proved in [54] that a stable domain is one-dimensional if and only if it is Mori. Since Mori domains satisfy the ascending chain condition on principal ideals, a Mori domain $D$ is Archimedean, that is $\bigcap_{n \geq 0} x^{n} D=(0)$, for each non-unit $x \in D$. The class of Archimedean domains includes also completely integrally closed domains and one-dimensional domains.

Problem 32a. Is a stable Archimedean domain one-dimensional?

The answer is positive in the semilocal case [54], so that a semilocal stable Archimedean domain is Mori (see also [49, Theorem 2.17]). Hence, a way of approaching this problem is trying to see if for stable domains the Archimedean property localizes. In general the Archimedean property does not pass to localizations. For example, the ring of entire functions is an infinite-dimensional completely integrally closed (hence, Archimedean) Bézout domain which is not locally Archimedean, because an Archimedean valuation domain is onedimensional. 
If $D$ is a Mori stable domain and $I$ is a nonzero ideal of $D$, we have $I^{v}=$ $(x, y)^{v}$, for some $x, y \in I$ [49, Theorem 2.17]. Therefore, we can say that in a stable Mori domain each divisorial ideal is 2-v-generated. Since a divisorial Mori domain is Noetherian, this result generalizes the fact that in a Noetherian domain that is stable and divisorial each ideal can be generated by two elements [49, Theorem 3.6].

Problem 32b. Let $D$ be a one-dimensional Mori domain such that each divisorial ideal is $2-v$-generated. Is it true that each divisorial ideal is stable?

Note that the answer to this question is negative when $D$ has dimension greater than one. For example, let $D$ be a Krull domain. Then, each divisorial ideal of $D$ is 2-v-generated [93, Proposition 1.2] and stability coincides with invertibility [49, Proposition 2.3]. Hence, each divisorial ideal of $D$ is stable (i.e., invertible) if and only if $D$ is locally factorial [17, Lemma 1.1].

Recall that a nonzero ideal $I$ of an integral domain $D$ is $v$-invertible if $(I(D$ : $I))^{v}=D$, and $I$ is called $v$-stable if $I^{v}$ is $v$-invertible as an ideal of $E\left(I^{v}\right)$, that is $\left(I^{v}\left(E\left(I^{v}\right): I^{v}\right)\right)^{v}=E\left(I^{v}\right)$. Clearly, $v$-invertibility implies $v$-stability. If each nonzero ideal of $D$ is $v$-stable, we say that $D$ is $v$-stable [52]. Each nonzero ideal of a Krull domain is $v$-invertible, thus a Krull domain is $v$-stable. However, a Krull domain is stable if and only if it is a Dedekind domain, that is, it has dimension one. An example of a one-dimensional Mori domain that is $v$-stable but not stable is given in [53, Example 2.6].

Problem 32c. Let $D$ be a Mori domain such that each divisorial ideal is 2 -v-generated. Is it true that $D$ is $v$-stable?

\section{Problem 33}

It is well known that if an integral domain $D$ has finite character, then a locally invertible ideal is invertible. Conversely, if each locally invertible ideal is invertible $D$ need not have finite character (for example, a Noetherian domain need not have finite character). However, a Prüfer domain such that each locally invertible ideal is invertible does have finite character. This fact was conjectured by S. Bazzoni [12, p 630] and proved by W. Holland, J. Martinez, W. McGovern and M. Tesemma in [76]. (A simplified proof appears in [91]). F. Halter-Koch gave independently another proof in the more general context of ideal systems [71]. Other contributions were made by M. Zafrullah in [111] and by C. A. Finocchiaro, G. Picozza and F. Tartarone in [41]. Following D. D. Anderson and M. Zafrullah [7], an integral domain $D$ is called an LPI-domain if each locally principal nonzero ideal of $D$ is invertible. Since a Prüfer domain is precisely a finitely stable integrally closed domain [49, Proposition 2.5], one is lead to ask the following more general question (which appears in [13, Question 4.6]):

Assume that $D$ is a finitely stable LPI-domain. Is it true that $D$ has finite character?

The answer to this question is positive if and only if the LPI-property extends to fractional overrings [50, Corollary 15]. In particular, this holds when $D$ is Mori or integrally closed. For an exhaustive discussion of this problem see [50]. 


\section{Problem 34}

Let $D$ be an integral domain, let $S$ be a multiplicatively closed set in $D$, and let $D_{S}$ be the ring of fractions of $D$ with respect to $S$. Consider $R=$ $D+X D_{S}[X]$, the ring of polynomials over $D_{S}$ in indeterminate $X$ and with constant terms in $D$.

Recall that a $G C D$ domain is an integral domain with the property that any two non-zero elements have a greatest common divisor; and a $P v M D$ is an integral domain with the property that every nonzero finitely generated ideal is $t$-invertible. It was shown in [26, Theorem 1.1] that $R$ is a GCD domain if and only if $D$ is a GCD domain and for all $d \in D \backslash\{0\} \operatorname{GCD}(d, X)$ exists. In [5, Theorem 2.5] it was shown that $R$ is a Prüfer $v$-multiplication domain (for short, $\mathrm{P} v \mathrm{MD})$ if and only if $D$ is a $\mathrm{P} v \mathrm{MD}$ and the ideal $(d, X)$ is $t$-invertible for all $d \in D \backslash\{0\}$. Next, recall that an integral domain is a $v$-domain if every nonzero finitely generated ideal is $v$-invertible. Note that if $D$ is a $v$-domain, it is possible that $D_{S}$ may not be a $v$-domain. For more information about $v$-domains consult [45].

Problem 34a. Find necessary and sufficient conditions for $R=D+$ $X D_{S}[X]$ to be a $v$-domain. Prove or disprove that $R$ is a $v$-domain if and only if $D$ is a $v$-domain and $(d, X)$ is $v$-invertible for all $d \in D \backslash\{0\}$.

More generally:

Problem 34b. Let $A \subseteq B$ be an extension of integral domains, and let $X$ be an indeterminate over $B$. Find necessary and sufficient conditions for the integral domain $A+X B[X]$ to be a $v$-domain.

\section{Problem 35}

An integral domain $D$ is called an almost $G C D$ (for short, AGCD) if for each pair $x, y \in D \backslash\{0\}$ there is an integer $n=n(x, y)$ (depending on $x$ and $y$ ) such that $x^{n} D \cap y^{n} D$ is principal. The theory of AGCD domains runs along lines similar to that of GCD domains; see [6] for more information and a list of references on the topic. An integral domain $D$ is a domain of finite $t$-character if every nonzero non-unit of $D$ belongs to at most a finite number of maximal $t$-ideals of $D$. AGCD domains of finite $t$-character were characterized in [34]. An ideal $A$ of $D$ is $t$-locally principal if $A D_{P}$ is principal for every maximal $t$-ideal $P$ of $D$. In [111] it was shown that if $D$ is a $\mathrm{P} v \mathrm{MD}$ then $D$ is of finite $t$-character if and only if every nonzero $t$-locally principal ideal of $D$ is $t$-invertible. A GCD domain is a $\mathrm{P} v \mathrm{MD}$ because the $v$-closure of every nonzero finitely generated ideal in a GCD domain is principal. We can therefore conclude that a GCD domain is of finite $t$-character if and only if every nonzero $t$-locally principal ideal $A$ of $D$ is $t$-invertible.

Now, we are in a position to state an open problem:

Let $D$ be an almost GCD domain such that every nonzero $t$-locally principal ideal is $t$-invertible. Is $D$ of finite $t$-character?

\section{Problem 36}


Let $D$ be an integral domain, let $K$ be the field of quotients of $D$ and let $\overline{\mathbf{F}}(D)$ denote the set of all the nonzero $D$-submodules of $K$.

Let $\mathcal{T}$ denote a non-empty collection of overrings of $D$ and, for any $T \in \mathcal{T}$, let $\star_{T}$ be a semistar operation on $T$. An interesting question posed in [23, Problem 44] is the following:

Problem 36a. Find conditions on $\mathcal{T}$ and on the semistar operations $\star_{T}$ under which the semistar operation $\star_{\mathcal{T}}$ on $D$ defined by $E^{\star} \mathcal{T}:=\bigcap\left\{(E T)^{\star_{T}} \mid T \in \mathcal{T}\right\}$, for all $E \in \overline{\boldsymbol{F}}(D)$, is of finite type.

Note that, if $D=\bigcap\{T \mid T \in \mathcal{T}\}$ is locally finite and each $\star_{T}$ is a star operation on $T$ of finite type, then D. D. Anderson in [3, Theorem 2] proved that $\star \mathcal{T}$ is a star operation on $D$ of finite type. Through the years, several partial answers to this question were given and they are mainly topological in nature. For example, in [43, Corollary 4.6], a description of when the semistar operation $\star_{\mathcal{T}}$ is of finite type was given when $\mathcal{T}$ is a family of localizations of $D$ and $\star_{T}$ is the identity semistar operation on $T$, for each $T \in \mathcal{T}$. More recently, in [40], it was proved that if $\mathcal{T}$ is a quasi-compact subspace of the (Zariski-Riemann) space of all valuation overrings of $D$ (endowed with the Zariski topology) and $\star_{T}$ is the identity (semi)star operation on $T$, for each $T \in \mathcal{T}$, then $\star_{\mathcal{T}}$ is of finite type. Another more natural way to see the problem stated above is the following.

Problem 36b. Let $\mathcal{S}$ be any non-empty collection of semistar operations on $D$ and let $\wedge_{\mathcal{S}}$ be the semistar operation defined by $E^{\wedge \mathcal{S}}:=\bigcap\left\{E^{\star} \mid \star \in \mathcal{S}\right\}$ for all $E \in \overline{\mathbf{F}}(D)$. Find conditions on the set $\mathcal{S}$ for the semistar operation $\wedge \mathcal{S}$ on $D$ to be of finite type.

Note that it is not so difficult to show that the constructions of the semistar operations of the type $\star \mathcal{T}$ and $\wedge_{\mathcal{S}}$ are essentially equivalent, in the sense that every semistar operation $\star \mathcal{T}$ can be interpreted as one of the type $\wedge_{\mathcal{S}}$, and conversely.

\section{Problem 37}

A finite-dimensional integral domain $D$ is said to be a Jaffard domain if $\operatorname{dim}\left(D\left[X_{1}, X_{2}, \ldots, X_{n}\right]\right)=n+\operatorname{dim}(D)$ for all $n \geq 1$; equivalently, if $\operatorname{dim}(D)=$ $\operatorname{dim}_{v}(D)$, where $\operatorname{dim}(D)$ denotes the (Krull) dimension of $D$ and $\operatorname{dim}_{v}(D)$ its valuative dimension (i.e., the supremum of dimensions of the valuation overrings of $D$ ). As this notion does not carry over to localizations, $D$ is said to be a locally Jaffard domain if $D_{P}$ is Jaffard for each prime ideal $P$ of $D$. The class of (locally) Jaffard domains contains most of the well-known classes of (locally) finite-dimensional rings involved in dimension theory such as Noetherian domains, Prüfer domains, universally catenarian domains, and universally strong $\mathrm{S}$ (eidenberg)-domains. It is an open problem to compute the dimension of polynomial rings over Krull domains in general. In this vein, Bouvier conjectured that "finite-dimensional Krull (or, more particularly, factorial) domains need not be Jaffard" [18, 44]. Bouvier's conjecture makes sense beyond the Noetherian context. Explicit finite-dimensional non-Noetherian Krull domains are scarce in the literature and one needs to test them and their localizations as well for 
the Jaffard property. In [16], the authors scanned all known families of examples of non-Noetherian finite dimensional Krull (or factorial) domains existing in the literature. They showed that all these examples - except two - are in fact locally Jaffard domains. The two exceptions are addressed below in the open problems $37 \mathrm{~b}$ and $37 \mathrm{c}$. Bouvier's conjecture is still elusively open and one may reformulate it in the following simple terms:

Problem 37a. Is there a Krull (or, more particularly, factorial) domain $D$ such that $1+\operatorname{dim}(D) \lesseqgtr \operatorname{dim}(D[X])$ ?

In [16], Bouchiba and Kabbaj examined David's second construction described in [29] of a 3-dimensional factorial domain which arises as an ascending union of 3-dimensional polynomial rings $J_{n}$ in three variables over a field $k$; namely, $J=\bigcup J_{n}$ with $J_{n}=k\left[X, \beta_{n-1}, \beta_{n}\right]$ for each positive integer $n$, where the variables $\beta_{n}$ satisfy the following condition: For $n \geq 2, \beta_{n}=\frac{-\beta_{n-1}^{s(n)}+\beta_{n-2}}{X}$, where the $s(n)$ are positive integers. We have $J_{n} \subseteq J \subseteq J_{n}\left[X^{-1}\right]$ for each positive integer $n$. Therefore, by [30, Theorem 2.3], $J$ is a Jaffard domain (since the $J_{n}$ 's are affine domains, i.e., finitely generated $k$-algebras), but it is not known if $J$ is locally Jaffard. So the following question is open:

Problem 37b. Is $J$ a locally Jaffard domain?

Clearly, a negative answer to problem 37b will solve (affirmatively) Bouvier's conjecture for factorial domains (cf. Problem 37a).

In [16], the authors also investigated the known family of examples which stem from the generalized fourteenth problem of Hilbert (also called HilbertZariski problem): Let $k$ be a field of characteristic zero, $T$ a normal affine domain (i.e., an integrally closed domain which is a finitely generated algebra) over $k$, and $F$ a subfield of the quotient field of $T$. The Hilbert-Zariski problem asks whether $D=F \cap T$ is an affine domain over $k$. Counterexamples for this problem were constructed by Rees [100], Nagata [94], and Roberts [101], where $D$ wasn't even Noetherian. In this vein, Anderson, Dobbs, Eakin, and Heinzer [9] asked whether $D$ and its localizations inherit from $T$ the Noetherian-like main behavior of having Krull and valuative dimensions coincide (i.e., whether $D$ is locally Jaffard). In [18], the authors proved that $D$ is Jaffard, but were not able to determine whether $D$ is locally Jaffard. In fact, they addressed this problem in the more general context of subalgebras of affine domains over Noetherian domains and the following question remains open:

Problem 37c. Let $A \subseteq D$ be an extension of integral domains, where $A$ is Noetherian domain and $D$ is a subalgebra of an affine domain $T$ over $A$. Is $D$ a locally Jaffard domain?

Clearly, a negative answer to Problem 37c will solve (affirmatively) Bouvier's conjecture for Krull domains.

\section{Problem 38}

A one-dimensional local Mori domain $R$ is called locally tame if for every irreducible element $u \in R$ there is a constant $t \in \mathbb{N}_{0}$ with the following property: For every $a \in u R$ and every factorization $v_{1} \cdot \ldots \cdot v_{m}$ of $a$ (where $m \in \mathbb{N}$ and 
$v_{1}, \ldots, v_{m}$ are irreducibles of $R$ ), there is a subproduct, say $v_{1} \cdot \ldots \cdot v_{l}$, which is a multiple of $u$ and which has a factorization containing $u$, say $v_{1} \cdot \ldots \cdot v_{l}=$ $u u_{2} \cdot \ldots \cdot u_{k}$ (where $k \in \mathbb{N}_{0}$ and $u_{2}, \ldots, u_{k}$ are irreducibles of $R$ ), such that $\max \{k, l\} \leq t$.

Let $R$ be a one-dimensional local Mori domain. Is $R$ locally tame?

Denote by $\widehat{R}$ the complete integral closure of $R$. It is known that $R$ is locally tame in each of the following cases: The conductor $(R: \widehat{R}) \neq\{0\}[56$, Proposition 2.10.7]; $R$ is Noetherian [73, Theorem 3.3]; $\widehat{R}$ is Krull and $|\mathfrak{X}(\widehat{R})| \geq 2$ [57, Theorem 3.5 and Corollary 3.6].

Assume that $R$ is locally tame. Then this implies the finiteness of several arithmetical invariants, such as the catenary degree and the set of distances of $R$. Also, the sets of lengths in $R$ have a well-defined structure [55, 58, and 56 , Theorem 3.1.1].

\section{Problem 39}

Analyze and describe non-unique factorization in $\operatorname{Int}(D)$, where $D$ is a DVR with finite residue field.

We remark that the results obtained by Frisch $[48]$ for $\operatorname{Int}(\mathbb{Z})$ rely heavily on the fact that $\mathbb{Z}$ has prime ideals of arbitrarily large index.

\section{Problem 40}

Let $\mathbb{Z}$ (respectively, $\mathbb{Q}$ ) denote the integers (respectively, the rationals), and let $F$ be a field. It is known that $\operatorname{Spec}(F[x, y])$ is order-isomorphic to $\operatorname{Spec}(\mathbb{Z}[y])$ if and only if $F$ is contained in the algebraic closure of a finite field [109, Theorem 2.10]; and in that case $\operatorname{Spec}(A)$ is order-isomorphic to $\operatorname{Spec}(\mathbb{Z}[y])$ for every twodimensional domain that is finitely generated as an $F$-algebra. Moreover, the poset $\operatorname{Spec}(\mathbb{Z}[y])$ is characterized, among posets, by five specific axioms $[21$, Theorem 2.9]. For more background information, see references [21, 108, 109, 110].

Problem 40a. Find axioms characterizing the poset $\mathbb{Q}[x, y]$.

See [21, Remark 2.11.3].

Problem 40b. Are $\operatorname{Spec}(\mathbb{Q}[x, y])$ and $\operatorname{Spec}(\mathbb{Q}(\sqrt{2})[x, y])$ order-isomorphic?

Problem 40c. More generally, let $F$ and $K$ be algebraic number fields. If $A$ is a two-dimensional affine domain over $F$, is $\operatorname{Spec}(A)$ order-isomorphic to $\operatorname{Spec}(K[x, y]) ?$

This question is close to Question 2.15.1 of [110]. It is also observed in Example 2.14 of [110], and Corollary 7 of [108], that, if $L$ is an algebraically closed field of infinite transcendence degree over $\mathbb{Q}$, then the spectra of $L[x, y, z] /\left(x^{4}+\right.$ $\left.y^{4}+z^{4}-1\right)$ and $L[x, y]$ are known to be non-isomorphic.

Problem 40d. At the other extreme, let $F$ and $K$ be fields, neither of them algebraic over a finite field. If $\operatorname{Spec}(F[x, y]$ and $\operatorname{Spec}(K[x, y])$ are orderisomorphic, are $F$ and $K$ necessarily isomorphic fields?

This is Question 2 of [110]. 


\section{Problem 41}

Let $I$ be the integral closure of the ideal $\left(x^{a}, y^{b}, z^{c}\right)$ in a polynomial ring in variables $x, y, z$ over a field.

Classify all triples $(a, b, c)$ for which all powers of $I$ are integrally closed.

For basic information on the integral closure of monomial ideals, see [81, Chapter 1, Section 1.4]. The case of two variables is known by Zariski's theory of integral closure of ideals in a two-dimensional regular ring [81, Chapter 14], and for three variables some work was done by Coughlin in [27].

\section{Problem 42}

For some special ideals, such as for monomial ideals, there are fast algorithms for computing their integral closure. For computing the integral closure of general ideals, however, the current algorithms reduce to computing the integral closure of the Rees algebra and then reading off the graded components of the integral closure This computes simultaneously the integral closures of all powers of the ideal, which is doing more than necessary. This excess of work makes the computation sometimes unwieldy.

Problem 42a. Is there a more direct algorithm for computing the integral closure of a general ideal?

Problem 42b. In particular, can the Leonard-Pelikaan [86] and SinghSwanson [104] algorithms be modified for computing the integral closure of ideals?

More information and references on the topic may be found in [105, Section $2]$.

\section{Problem 43}

The following is a general form of the Lipman-Sathaye Theorem, as found in Theorem 2.1 of [75]: Let $R$ be a Noetherian domain with field of fractions $K$. Assume that the $S_{2}$-locus is open in all algebras essentially of finite type over $R$. Let $S$ be an extension algebra essentially of finite type over $R$ such that $S$ is torsion-free and generically étale over $R$, and such that for every maximal ideal $M$ of $S, R_{M \cap R}$ is normal, and $S_{M}$ has a relatively $S_{2}$-presentation over $R_{M \cap R}$. Let $L=K \otimes_{R} S$ and let $\bar{S}$ be the integral closure of $S$ in L. Assume that $\bar{S}$ is module-finite over $S$, and that for every height-one prime ideal $Q$ of $\bar{S}, R_{Q \cap R}$ is regular. Then $\left(\bar{S}:_{L} J_{\bar{S} / R}\right) \subseteq\left(S:_{L} J_{S / R}\right)$.

The suggested problem is to find how tight is the statement of this theorem: relax some assumption and either prove the theorem or find a counterexample for that relaxation.

\section{Problem 44}

Let $R$ be a ring and let $I$ be an ideal in $R$. A set of Rees valuation rings of $I$ is a set $\left\{V_{1}, . ., V_{r}\right\}$ consisting of valuation rings, subject to the following conditions:

(1) Each $V_{i}$ is Noetherian and is not a field. 
(2) For each $i=1, \ldots, r$ there exists a minimal prime ideal $P_{i}$ of $R$ such that $V_{i}$ is a ring between $R / P_{i}$ and the field of fractions of $R / P_{i}$.

(3) For each natural number $n, \overline{I^{n}}=\cap_{i=1}^{r}\left(I^{n} V_{i}\right) \cap R$, where $\overline{I^{n}}$ denotes the integral closure of $I^{n}$.

(4) No set of valuation rings of cardinality smaller than $r$ satisfies conditions (1) $-(3)$.

Basic information on Rees valuations of ideals may be found in [81, Chapter 10]. Ideals that have only one Rees valuation have several good properties.

Cutkosky proved in [28] the existence of a Noetherian, two-dimensional, complete, integrally closed local domain $(R, m)$ in which every $m$-primary ideal has more than one Rees valuation. Give a construction of such a ring.

\section{Acknowledgements}

We thank all the commutative algebraists who contributed open problems to this article. The list of contributors is as follows: D. D. Anderson (problem 8), A. Badawi (problem 30), P.-J. Cahen (problems 14 and 15), J.-L. Chabert (problems 16, 17, and 18), J. Elliott (problems 19, 20, 21, 22, and 23), C. A. Finocchiaro and M. Fontana (problem 36), S. Frisch (problems 28 and 39), S. Gabelli (problems 32 and 33), A. Geroldinger (problem 38), S. Glaz (problems 1, 2, and 3), L. Hummel (problem 7), K. Johnson (problems 24 and 25), S. Kabbaj (problem 37), T. G. Lucas (problems 9, 10, 11, and 12), B. Olberding (problems 29 and 31), G. Peruginelli (problem 26), G. Picavet and M. Picavet-L'Hermitte (problem 13), R. Schwarz (problems 4, 5, and 6), I. Swanson (problems 41, 42, 43, and 44), N. J. Werner (problems 27 and 28), S. Wiegand and R. Wiegand (problem 40), M. Zafrullah (problems 34 and 35).

\section{References}

[1] D. Adam, J.-L. Chabert and Y. Fares, Subsets of $Z$ with simultaneous ordering, Integers 10 (2010), 437-451.

[2] T. Akiba, Integrally-closedness of polynomial rings, Japan. J. Math., 6 (1980), 67-75.

[3] D. D. Anderson, Star operations induced by overrings, Comm. Algebra 16 (1988), 2535-2553.

[4] D. D. Anderson, Quasi-complete semilocal rings and modules, this volume.

[5] D. D. Anderson, D. F. Anderson and M. Zafrullah, The ring $D+$ $X D_{S}[X]$ and t-splitting sets, Arab. J. Sci. Eng., Sect. C, Theme Issues 26 (1): Commutative Algebra (2001), 3-16.

[6] D. D. Anderson and M. Zafrullah, Almost Bézout domains, J. Algebra 142 (1991), 285-309.

[7] D. D. Anderson and M. Zafrullah, Integral domains in which nonzero locally principal ideals are invertible, Comm. Algebra 39 (2011), 933-941.

[8] D. F. Anderson and A. Badawi, On n-absorbing ideals of commutative rings, Comm. Algebra 39, (2011), 1646-1672. 
[9] D. F. Anderson, D. E. Dobbs, P. M. Eakin, and W. J. Heinzer, On the generalized principal ideal theorem and Krull domains, Pacific J. Math. 146 (2) (1990), 201-215.

[10] M. André, Non-Noetherian complete intersections, Bull. Amer. Math. Soc. 78 (1972), 724-729.

[11] A. Badawi, On 2-absorbing ideals of commutative rings, Bull. Austral. Math. Soc. 75 (2007), 417-429.

[12] S. Bazzoni, Class semigroups of Prüfer domains, J. Algebra 184 (1996), 613-631.

[13] S. Bazzoni, Finite character of finitely stable domains, J. Pure Appl. Algebra 215 (2011), 1127-1132.

[14] S. Bazzoni \& S. Glaz, Gaussian properties of total rings of quotients, J. Algebra 310 (2007), 180-193.

[15] M. Bhargava, The factorial function and generalizations, Amer. Math. Monthly, 107 (2000), 783-799.

[16] S. Bouchiba and S. Kabbaj, Bouvier's conjecture, Commutative Algebra and its Applications, de Gruyter, Berlin, 2009, 79-88.

[17] A. Bouvier, The local class group of a Krull domain, Canad. Math. Bull. 26 (1983), 13-19.

[18] A. Bouvier and S. Kabbaj, Examples of Jaffard domains, J. Pure Appl. Algebra 54 (2-3) (1988), 155-165.

[19] P.-J. Cahen and J.-L. Chabert, Integer-Valued Polynomials. Amer. Math. Soc. Surveys and Monographs, 48, Providence, 1997.

[20] P.-J. Cahen and R. Rissner, Finiteness and Skolem closure of ideals for non unibranched domains, submitted.

[21] E. Celikbas, C. Eubanks-Turner and S. Wiegand, Prime ideals in polynomial and power series rings over Noetherian domains, this volume.

[22] J.-L. Chabert, Integer-valued polynomials: Looking for regular bases (a survey), this volume.

[23] S. Chapman and S. Glaz, One hundred problems in commutative ring theory, Non-Noetherian Commutative Ring Theory, Math. Appl., 520, Kluwer Acad. Publ., Dordrecht, 2000, 459-476.

[24] C. Chevalley, On the theory of local rings, Ann. Math. 44 (1943), $690-708$.

[25] Z. Coelho and W. Parry, Ergodicity of p-adic multiplications and the distribution of Fibonacci numbers, Amer. Math. Soc. Translations 202 (2001), 51-70.

[26] D. Costa, J. L. Mott and M. Zafrullah, The construction $D+X D_{S}[X]$, J. Algebra 53 (1978), 423-439.

[27] H. Coughlin, Classes of Normal Monomial Ideals, Ph. D. thesis, University of Oregon, 2004.

[28] S. D. Cutkosky, On unique and almost unique factorization of complete ideals II, Invent. Math. 98 (1989), 59-74.

[29] J. David, A characteristic zero non-Noetherian factorial ring of dimension three, Trans. Amer. Math. Soc. 180 (1973), 315-325. 
[30] D. E. Dobbs, M. Fontana and S. Kabbaj, Direct limits of Jaffard domains and S-domains, Comment. Math. Univ. St. Pauli 39 (2) (1990), $143-155$.

[31] D. Dobbs and G. Picavet, Straight rings. Comm. Algebra 37 (3) (2009), 757-793.

[32] D. Dobbs, G. Picavet and M. Picavet-L'Hermitte, On a new class of integral domains with the portable property, this volume.

[33] A. Douglas, The weak global dimension of the group rings of abelian groups, J. London Math. Soc. 36 (1961), 371-381.

[34] T. Dumitrescu, Y. Lequain, J. Mott and M. Zafrullah, Almost GCD domains of finite t-character, J. Algebra 245 (2001), 161-181.

[35] J. Elliott, Universal properties of integer-valued polynomial rings, J. Algebra 318 (2007) 68-92.

[36] J. Elliott, Birings and plethories of integer-valued polynomials, Third International Meeting on Integer-Valued Polynomials (2010), Actes des Rencontres du CIRM 2 (2) (2010), 53-58.

[37] J. Elliott, Integer-valued polynomial rings, t-closure, and associated primes, Comm. Algebra 39 (11) (2011), 4128-4147.

[38] J. Elliott, The probability that $\operatorname{Int}_{n}(D)$ is free, in this volume.

[39] A. Fabbri and O. Heubo-Kwegna, Projective star operations on polynomial rings over a field, J. Commut. Algebra 4 (3) (2012), 387-412.

[40] C. A. Finocchiaro, M. Fontana and K. A. Loper, The constructible topology on spaces of valuation domains, Trans. Amer. Math. Soc. 365 (12) (2013), 6199-6216.

[41] C. A. Finocchiaro, G. Picozza and F. Tartarone, Star-invertibility and $t$-finite character in integral domains, J. Algebra Appl. 10 (2011), 755-769.

[42] M. Fontana, E. Houston and T. G. Lucas,Toward a classification of prime Ideals in Prüfer domains, Forum Mathematicum, 22 (2010), 741-766.

[43] M. Fontana and J. Huckaba, Localizing systems and semistar operations, Non-Noetherian Commutative Ring Theory, Math. Appl., 520, Kluwer Acad. Publ., Dordrecht, 2000, 169-197.

[44] M. Fontana and S. Kabbaj, Essential domains and two conjectures in dimension theory, Proc. Amer. Math. Soc. 132 (2004), 2529-2535.

[45] M. Fontana and M. Zafrullah, On v-domains: A survey, Commutative Algebra: Noetherian and Non-Noetherian Perspectives, Springer, New York, 2011, 145-179.

[46] S. Frisch, Polynomial separation of points in algebras, Arithmetical Properties of Commutative Rings and Modules (Chapel Hill Conference Proceedings.), Dekker 2005, 249-254.

[47] S. Frisch, Integer-valued polynomials on algebras, J. Algebra 373 (2013), 414-425. Corrigendum to: Integer-valued polynomials on algebras [J. Algebra 373 (2013), 414-425], J. Algebra, in press. http://dx.doi.org/10.1016/j.jalgebra.2013.06.003.

[48] S. Frisch, A Construction of integer-valued polynomials with prescribed sets of lengths of factorizations, Monatsh. Math. 171 (3-4) (2013), 341-350.

[49] S. Gabelli, Ten problems on stability of domains, this volume. 
[50] S. Gabelli, Locally principal ideals and finite character, Bull. Math. Soc. Sci. Math. Roumanie (N.S.), Tome 56 (104) (2013), 99-108.

[51] S. Gabelli and E. Houston, Coherent-like conditions in pullbacks, Michigan Math. J. 44 (1997), 99-123.

[52] S. Gabelli and G. Picozza, Star-stable domains, J. Pure Appl. Algebra 208 (2007), 853-866.

[53] S. Gabelli and G. Picozza, Star stability and star regularity for Mori domains, Rend. Semin. Mat. Padova, 126 (2011), 107-125.

[54] S. Gabelli and M. Roitman, On finitely stable domains, preprint.

[55] W. Gao, A. Geroldinger, and W. A. Schmid, Local and global tameness in Krull monoids, Comm. Algebra, to appear.

[56] A. Geroldinger and F. Halter-Koch, Non-Unique Factorizations:.Algebraic, Combinatorial and Analytic Theory, Pure and Applied Mathematics 278, Chapman \& Hall/CRC Press, 2006.

[57] A. Geroldinger, W. Hassler, and G. Lettl, On the arithmetic of strongly primary monoids, Semigroup Forum 75 (2007), 567-587.

[58] A. Geroldinger and W. Hassler, Local tameness of v-Noetherian monoids, J. Pure Appl. Algebra 212 (2008), 1509-1524.

[59] R. Gilmer, Multiplicative Ideal Theory, Marcel Dekker, New York, 1972.

[60] R. Gilmer, Commutative Semigroup Rings, Chicago Lecture Notes in Mathematics, University of Chicago Press, Chicago, 1984.

[61] R. Gilmer and T. Parker, Divisibility properties in semigroup rings, Mich. Math. J. 21 (1974), 65-86.

[62] S. Glaz, On the weak dimension of coherent group rings, Comm. Algebra. 15 (1987), 1841-1858.

[63] S. Glaz, Commutative Coherent Rings, Lecture Notes in Mathematics 1371. Springer-Verlag, Berlin, 1989.

[64] S. Glaz, Finite conductor rings, Proc. Amer. Math. Soc. 129 (2000), 2833-2843.

[65] S. Glaz, Finite conductor rings with zero divisors, Non-Noetherian Commutative Ring Theory, MAIA 520, Kluwer Acad. Publ., Dordrecht, 2000, 251270 .

[66] S. Glaz, Prüfer conditions in rings with zero-divisors, Lecture Notes in Pure Appl. Math. 241, CRC Press, London, 2005, 272-281.

[67] S. Glaz, The weak dimension of Gaussian rings, Proc. Amer. Math. Soc. 133 (2005), 2507-2513.

[68] S. Glaz and R. Schwarz, Prüfer conditions in commutative rings, Arabian J. Sci.\& Eng. 36, (2011), 967-983.

[69] S. Glaz and R. Schwarz, Finiteness and homological conditions in commutative group rings, Progress in Commutative Algebra 2, De Gruyter, 2012, 129-143.

[70] F. Halter-Koch, Kronecker function rings and generalized integral closures, Comm. Algebra 31 (2003), 45-59.

[71] F. Halter-Koch, Clifford semigroups of ideals in monoids and domains, Forum Math. 21 (2009), 1001-1020. 
[72] T. Hamilton and T. Marley, Non-Noetherian Cohen-Macaulay rings, J. Algebra 307 (2007), 343-360.

[73] W. Hassler, Arithmetical properties of one-dimensional, analytically ramified local domains, J. Algebra 250 (2002), 517-532.

[74] W. Heinzer, C. Rotthaus and S. Wiegand, Examples using power series over Noetherian integral domains, in preparation

(http://www.math.purdue.edu/ heinzer/preprints/preprints.html)

[75] M. Hochster, Presentation depth and the Lipman-Sathaye Jacobian theorem, The Roos Festschrift, Vol.2: Homology Homotopy Appl. 4 (2002), 295314 .

[76] W. C. Holland, J. Martinez, W. Wm. McGovern and M. Tesemma, Bazzoni's conjecture, J. Algebra 320 (4) (2008), 1764-1768.

[77] E. Houston and M. Zafrullah, Integral domains in which each t-ideal is divisorial, Michigan Math J. 35 (2), (1988), 291-300.

[78] J. A. Huckaba, Commutative Rings with Zero Divisors, Dekker, New York, 1988.

[79] L. Hummel, Recent progress in coherent rings: a homological perspective, Progress in Commutative Algebra 1, De Gruyter, 2012, 271-292.

[80] L. Hummel and T. Marley, The Auslander-Bridger formula and the Gorenstein property for coherent rings, J. Commutative Alg. 1 (2009), 283314.

[81] C. Huneke and I. Swanson, Integral Closure of Ideals, Rings, and Modules, London Mathematical Society Lecture Note Series, 336. Cambridge University Press, Cambridge, 2006.

[82] C. J. Hwang and G. W. Chang, Prüfer v-multiplication domains in which each t-ideal is divisorial, Bull. Korean Math. Soc. 35 (2) (1998), 259268.

[83] K. Johnson and K. Scheibelhut, Polynomials that are integer valued on the Fibonacci numbers, to appear.

[84] G. Karpilovsky, Commutative Group Algebras, Lecture Notes in Pure Appl. Math 78, Marcel Dekker, New York, 1983.

[85] T. Y. Lam, A First Course in Non-commutative Rings, Springer, New York, 1991.

[86] D. A. Leonard and R. Pellikaan, Integral closures and weight functions over finite fields, Finite Fields Appl. 9 (2003), 479-504.

[87] A. K. Loper, A classification of all D such that Int $(D)$ is a Prüfer domain, Proc. Amer. Math Soc. 126 (3) (1998), 657-660.

[88] K. A. Loper and F. Tartarone, A classification of the integrally closed rings of polynomials containing $Z[X]$, J. Commut. Algebra 1 (1) (2009), 91157.

[89] K. A. Loper and N.J. Werner, Generalized rings of integer-valued polynomials, J. Number Theory 132 (11) (2012), 2481-2490.

[90] T. G. Lucas, Two annihilator conditions: Property (A) and (a.c.), Comm. Algebra 14 (1986), 557-580.

[91] W. Wm. McGovern, Prüfer domains with Clifford class semigroup, J. Commut. Algebra 3 (2011), 551-559. 
[92] A. Mingarelli, Abstract factorials, arXiv:0705.4299v3 [math.NT] $10 \mathrm{Jul}$ 2012.

[93] J. L. Mott and M. Zafrullah, On Krull domains, Arch. Math. 56 (1991), 559-568.

[94] M. Nagata, On the fourteenth problem of Hilbert, Proceedings of the International Congress of Mathematicians, 1958, Cambridge University Press, London-New York, 1960,.459-462.

[95] A. Okabe and R. Matsuda, Semistar operations on integral domains, Math. J. Toyama Univ. 17 (1994), 1-21.

[96] B. Olberding, Intersections of valuation overrings of two-dimensional Noetherian domains, Commutative Algebra-Noetherian and Non-Noetherian Perspectives, Springer, New York, 2011, 459-462.

[97] B. Osofsky, Global dimensions of commutative rings with linearly ordered ideals, J. Lond. Math. Soc. 44 (1969), 183-185.

[98] G. Peruginelli, Integer-valued polynomials over matrices and divided differences, to appear in Monatshefte für Mathematik

(online at: http://dx.doi.org/10.1007/s00605-013-0519-9)

[99] G. Peruginelli and N.J. Werner, Integral closure of rings of integervalued polynomials on algebras, this volume.

[100] D. Rees, On a problem of Zariski, Illinois J. Math. 2 (1958), 145-149.

[101] P. Roberts, An infinitely generated symbolic blow-up in a power series ring and a new counterexample to Hilbert's fourteenth problem, J. Algebra 132 (1990), 461-473.

[102] K. Scheibelhut, Polynomials that are integer valued on the Fibonacci numbers, Msc. Thesis, Dalhousie University, Aug. 2013.

[103] R. Schwarz and S. Glaz, Commutative group rings with von Neumann regular total rings of quotients, J. Algebra 388 (2013), 287-293.

[104] A. K. Singh and I. Swanson, Associated primes of local cohomology modules and of Frobenius powers, International Mathematics Research Notices 30 (2004), 1703-1733.

[105] I. Swanson, Integral Closure, this volume.

[106] N.J. Werner, Integer-valued polynomials over matrix rings, Comm. Algebra 40 (12) (2012), 4717-4726.

[107] N. J. Werner, Polynomials that kill each element of a finite ring, to appear in J. Algebra Appl.

[108] R. Wiegand, The prime spectrum of a two-dimensional affine domain, J. Pure Appl. Algebra 40 (1986), 209-214.

[109] R. Wiegand and S. Wiegand, Prime ideals and decompositions of modules, Non-Noetherian Commutative Ring Theory, Math. Appl., 520, Kluwer Acad. Publ., Dordrecht, 2000, 403-428.

[110] R. Wiegand and S. Wiegand, Prime ideals in Noetherian rings: A survey, Ring and Module Theory, Birkhauser, Boston, MA, 2010, 175-193.

[111] M. Zafrullah, t-invertibility and Bazzoni-like statements, J. Pure Appl. Algebra 214 (2010), 654-657. 